\title{
B4GALT7-related spondylodysplastic Ehlers-Danlos syndrome
}

INSERM

\section{Source}

INSERM. (1999). Orphanet: an online rare disease and orphan drug data base. B4GALT7related spondylodysplastic Ehlers-Danlos syndrome. ORPHA:75496

Ehlers-Danlos syndrome, progeroid type (EDS-PF) is a form of Ehlers-Danlos syndrome characterized by a premature aging with sparse hair, macrocephaly, loose elastic skin, failure to thrive, joint laxity, psychomotor retardation, hypotonia, and defective wound healing with atrophic scars. 